Volume 19, No 3 International Journal of Radiation Research, July 2021

\title{
Analysis of size-specific dose profiles beyond the scan range in water phantoms undergoing computed tomography examinations
}

\author{
M. Masoudi ${ }^{1}$, R. Faghihi ${ }^{1,2^{*}}$, S. Sina ${ }^{1,2}$ \\ ${ }^{1}$ Nuclear Engineering Department, Shiraz University, Shiraz, Iran \\ ${ }^{2}$ Radiation Research Center, Shiraz University, Shiraz, Iran
}

\section{- Original article}

\begin{tabular}{|c|}
\hline $\begin{array}{l}\text { *Corresponding authors: } \\
\text { Reza Faghihi, Ph.D., } \\
\text { E-mail: } \\
\quad \text { faghihir@shirazu.ac.ir }\end{array}$ \\
\hline $\begin{array}{l}\text { Revised: July } 2020 \\
\text { Accepted: July } 2020\end{array}$ \\
\hline $\begin{array}{l}\text { Int. J. Radiat. Res., July 2021; } \\
\text { 19(3): 551-557 }\end{array}$ \\
\hline DOI: $10.29252 /$ ijrr.19.2.55 \\
\hline
\end{tabular}

\begin{abstract}
Background: The purpose of this study is to analyze the cumulative dose profile tail beyond the scan lengths of $10-90 \mathrm{~cm}$ in CT examinations on central and peripheral axes of water phantoms $(10-50 \mathrm{~cm}$ diameter). The analysis includes extension, trend, and dependency of the tails on the scan length and phantom diameter. Materials and Methods: A validated Monte Carlo model of a GE LightSpeed 16-slice CT scanner was used to obtain a dose profile from narrow beam collimation $(1 \mu \mathrm{m})$. The cumulative dose profile tail was developed by applying the well-known "convolution method", i.e. convolving a dose profile with a RECT function. The tails were analyzed according to the phantom diameter and scan length statistically. Results: For all phantoms, the statistical analysis shows no significant differences between tails of scan lengths of $10-90 \mathrm{~cm}$ for peripheral axis and those of scan lengths $>30 \mathrm{~cm}$ for the central axis. The tails have an exponential falloff from the scan edge modeled by $D(z)=A e^{-B z}$. The $A$ and $B$ are related to the falloff speed and amplitude of the tails. By increasing the phantom diameter, the amplitude and falloff speed decrease $82 \%$ and $59 \%$ on the central axis and $65 \%$ and $31 \%$ on the peripheral axis respectively. Conclusion: A simple equation was suggested for dose calculation at any point beyond the scan range on the central and peripheral axes of water phantoms. The equation is independent of scan length and is useful for evaluating the dose of organs located at the edge or outside of the scan region.
\end{abstract}

Keywords: Computed tomography, dose profile, GATE simulation, cumulative dose.

\section{INTRODUCTION}

Radiation dose in the Computed Tomography (CT) examinations is involved by rotational irradiation geometry, different scanning exposure settings, and patients with different sizes and body habits (1). The radiation dose in CT examinations for patients with different sizes has been studied in two ways including the direct organ dose assessment and the so-called "CT dose index (CTDI)" methodology (2-5).

The second way is the base of a quick CT dose evaluation before exposure by displaying the volume CT dose index (CTDI $\left.{ }_{\mathrm{vol}}\right)$ and dose length product (DLP) on the CT scanner consoles for 32 $\mathrm{cm}$ and $16 \mathrm{~cm}$ poly-methyl-methacrylate (PMMA) phantoms ${ }^{(6)}$.

The CTDI-based methodology (using cylindrical phantoms) suffers from some aspects such as (1) difference between the patient size and CTDI phantoms, (2) low length of the CTDI phantoms $(15 \mathrm{~cm})$ and (3) inadequate integral length of dose profile along the z-axis $(10 \mathrm{~cm})$ for capturing whole scatter tails of the dose profiles (3-4). The new dose quantities introduced to overcome these limitations are "size-specific dose estimate (SSDE)" (for considering patient size differences) and "cumulative dose" (for 
capturing scatter tails of dose profile) $(7,8)$.

The SSDE for each patient is calculated by applying the size correction factors to the $\mathrm{CTDI}_{\mathrm{vol}}{ }^{(8)}$. On the other hand, the cumulative dose is obtained by a so-called "convolution method" in which a single slice dose profile on the central or peripheral axis is convolved by a RECT function. The RECT function may represent the primary beam width or scan length in the convolution process (2).

Some investigations have been performed on the cumulative dose profile, equilibrium dose (i.e. cumulative dose at the center of enough long scan length), approach to equilibrium function (the cumulative dose dependency on the scan length) and the longitudinal distribution of the cumulative dose profile for different CT scanner models and exposure settings, scan lengths and also phantom composition and sizes (2,9-11). Also, some mathematical equations have been proposed for the cumulative dose profile description (9-11). An interesting result reported in such studies was that the cumulative dose profile has a long tail beyond the scan range which is responsible for the dose of organs located outside the scan region in a typical CT examination.

However, the detailed analysis of this tail has not been the purpose of the investigations performed. Therefore, the present study aimed to analyze size-specific cumulative dose profiles beyond the scan range in water phantoms and propose a simple framework for quick calculation of dose at any point beyond the scan range along the z-axis.

\section{MATERIALS AND METHODS}

Two main steps performed in this study are: modeling and validating a GE LightSpeed 16 slice CT scanner by using the GATE Monte Carlo toolkit and determining the cumulative dose profile tail for different water cylindrical phantoms by applying the convolution method.

\section{CT scanner and phantom modeling}

A GE LightSpeed 16 slice CT scanner (General Electric Medical Systems, Waukesha, WI) was 552 modeled by the GATE V8.0 code which is a powerful tool for simulating a CT source rotating around the object and acquiring data dynamically $(12,13)$. The CT scanner model consists of a point source rotating on a circular path with the center of isocenter and radius of focus-isocenter distance, i.e. $541 \mathrm{~mm}$. The model takes into account X-ray source and spectra as well as flat and bowtie filters geometry. The accurate modeling of the CT X-ray source requires obtaining the $\mathrm{X}$-ray spectra, collimating the beam, and moving the source around the isocenter.

To obtain the initial X-ray spectra for the tube voltages of 80,100 and $120 \mathrm{kVp}$, Institute of Physics and Engineering in Medicine Report 78 program (Srs-78 program) was used (14) with known CT scanner anode (tungsten with the angle of $7^{\circ}$ ) and filters (flat and bowtie filters). The X-ray spectra were collimated according to the head and body scan field of views and 10 $\mathrm{mm}$ beam collimation at the isocenter. The source was attached to an arbitrary geometrical volume and was rotated in the circular path to define a total of 100 projections in one slice. A total of $10^{8}$ photons were emitted toward the isocenter in one slice (15).

The standard head and body CT dosimetry cylindrical phantoms $(15 \mathrm{~cm}$ long, 16 , and $32 \mathrm{~cm}$ diameters, PMMA with the density of $1.195 \mathrm{gr} /$ $\mathrm{cm}^{3}$ ) were simulated along with their central and peripheral holes $(12.4 \mathrm{~mm}$ diameter, 100 $\mathrm{mm}$ long) for CTDI calculation. The geometry of the CT scanner model and phantoms are shown in figure 1.

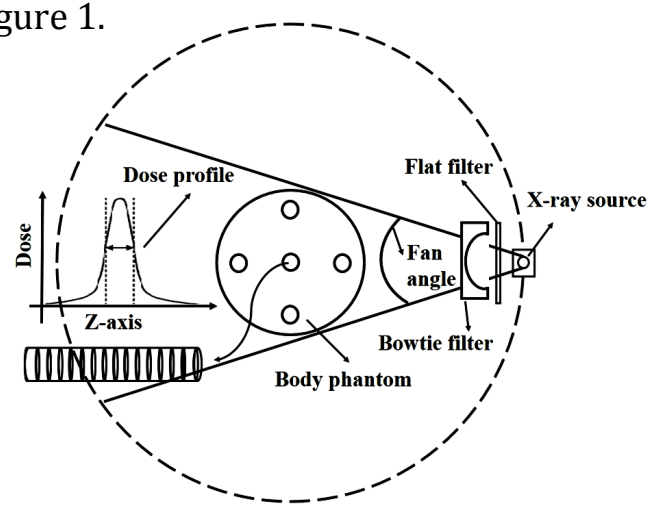

Figure 1. The geometry of the CT scanner and phantom model including the X-ray source and its fan angle for indicating the scan field of view, flat and bowtie filters, body phantom and its holes, and a typical dose profile for the central axis of the body phantom.

Int. J. Radiat. Res., Vol. 19 No. 3, July 2021 
The output of the simulated model is the energy deposited in each hole. Therefore, the

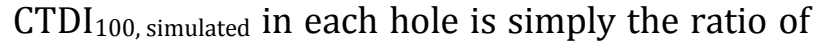
the total energy deposited in each hole to the mass of the hole. Then, the real CTDI value is the CTDI $_{100, \text { simulated multiplied by a normalization }}$ factor (NF) and mAs. The NF (particle/ mAs) calibrates the number of required photons at each $\mathrm{kVp}$ for simulation of $1 \mathrm{mAs}(16,17)$. The real CTDI values calculated from the MC model were compared to the corresponding measured CTDI values for validation.

\section{Cumulative dose profile tail determination}

The convolution method was applied to determine a cumulative dose profile for a series of contiguous axial CT scans on the central and peripheral axes. In the convolution method, the so-called "dose spread function (DSF)" is convolved by a RECT function. The DSF is a dose profile obtained from a narrow mathematical primary fan beam in a given exposure condition. In this study, the exposure conditions in the MC model were the body bowtie filter, $100 \mathrm{mAs}, 120$ $\mathrm{kVp}$, and $1 \mu \mathrm{m}$ narrow fan beam collimation (4). The $90 \mathrm{~cm}$ long water phantoms with diameters ranging from $10-50 \mathrm{~cm}$ were modeled for the fully capturing of scattering tails in the phantoms (11). The central and peripheral holes of the phantoms were divided into $1 \mathrm{~mm}$ intervals in the longitudinal direction to calculate the dose in each interval and obtain the DSFs as shown in figure 1. The RECT function is obtained from equation $1{ }^{(2)}$.

$$
\begin{array}{ll}
\Pi(z)=P_{0} & -\frac{L}{2} \leq z \leq \frac{L}{2} \\
\Pi(z)=0 & \text { elsewhere }
\end{array}
$$

Where; $\mathrm{P}_{0}$ is a constant value and $\mathrm{L}$ is the scan length (ranging from $10-90 \mathrm{~cm}$ in this study). The cumulative dose profile tail beyond the scan range was extracted from the cumulative dose profile and analyzed as reported in the results section.

\section{Statistical analysis}

The statistical analyses were performed using "OriginPro software" (version 9.6, OriginLab
Corporation, USA). This software is a data analysis and graphing software with the ability of statistical analysis of data. First, the "Shapiro-Wilk test" was used for testing the normality of the data. Accordingly, the "t-test" (for comparing two data sets) and the "ANOVA one-way test" (for comparing more than two data sets) were applied for data with normal distribution (18).

\section{RESULTS}

\section{Validation of the CT scanner model}

Table 1 shows the $\mathrm{CTDI}_{100}$ values obtained from the MC model and measurement on the central and peripheral axes of the head and body dosimetry phantoms. The paired t-test analysis shows that the simulated and measured data have no significant difference statistically (average difference of the two sets of data is < $5.5 \%$ ) which validates the CT scanner model for more investigations.

\section{DSF scatter component}

As stated in the materials and method section, the DSF profiles are the base of the convolution method. In this section, the scatter component of the DSFs was analyzed since this component has an important effect on the cumulative dose profile tail (4). Figure 2 shows the normalized scatter component of DSF for the $16 \mathrm{~cm}$ and $45 \mathrm{~cm}$ water phantoms on the central and peripheral axes.

\section{Cumulative dose profile tail beyond the scan range}

Figure $3(a, b)$ shows the cumulative dose profile tails beyond the different scan lengths $(10-90 \mathrm{~cm})$ on the central axis for the $20 \mathrm{~cm}$ and $50 \mathrm{~cm}$ phantoms. Figure 4 shows the cumulative dose profile tails beyond different scan lengths $(10-90 \mathrm{~cm})$ on the peripheral axis for the $50 \mathrm{~cm}$ phantom. For the small phantoms, the cumulative dose profile tails have a trend similar to the large phantoms for which the data is not shown here. 
Table 1. The CTDI100 (mGy) values obtained from the MC model and measurement.

\begin{tabular}{|c|c|c|c|c|c|c|c|}
\hline \multirow{2}{*}{ kVp } & \multirow{2}{*}{ Position } & \multicolumn{2}{|c|}{ Head phantom } & \multirow{2}{*}{ Difference \% } & \multicolumn{2}{|c|}{ Body phantom } & \multirow{2}{*}{ Difference \% } \\
\cline { 3 - 7 } & & Measured & Simulated & & Measured & Simulated & \\
\hline \multirow{2}{*}{$\mathbf{8 0}$} & Center & 7.4 & 7.5 & 1.76 & 1.8 & 1.9 & 7.78 \\
\cline { 2 - 7 } & Periphery & 7.6 & 7.9 & 3.42 & 3.9 & 4.1 & 4.62 \\
\hline \multirow{2}{*}{$\mathbf{1 0 0}$} & Center & 13.8 & 14.4 & 4.57 & 4.3 & 4.6 & 6.74 \\
\cline { 2 - 7 } & Periphery & 13.7 & 14.1 & 3.07 & 8.3 & 9.1 & 9.40 \\
\hline \multirow{2}{*}{$\mathbf{1 2 0}$} & Center & 21.2 & 21.6 & 2.12 & 7.2 & 7.0 & 3.19 \\
\cline { 2 - 7 } & Periphery & 20.7 & 20.9 & 0.77 & 13.9 & 14.0 & 1.15 \\
\hline
\end{tabular}

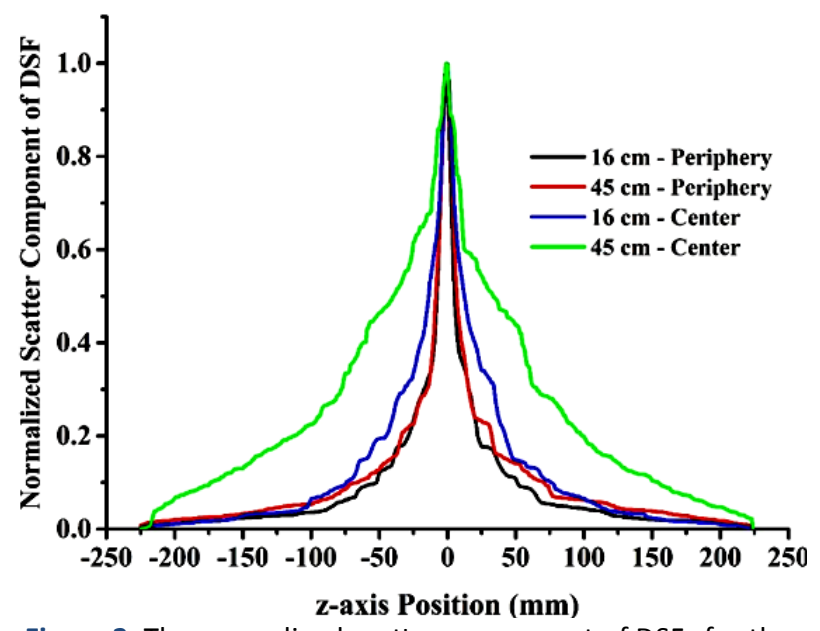

Figure 2. The normalized scatter component of DSFs for the 16 and $45 \mathrm{~cm}$ water phantoms on the central and peripheral axes.

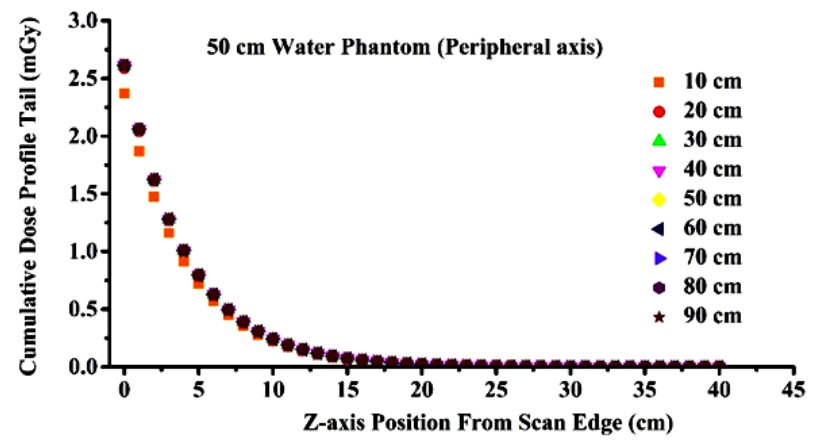

Figure 4. Cumulative dose profile tails beyond the scan lengths of $10-90 \mathrm{~cm}$ on the peripheral axis for $50 \mathrm{~cm}$ water phantom.

As can be seen, the cumulative dose profile tails beyond the scan range have a relatively similar extension and trend for almost all scan lengths $(10-90 \mathrm{~cm})$ on the central and peripheral axes. The ANOVA one-way test shows no statistically significant differences between cumulative dose profile tails of different scan lengths $(10-90 \mathrm{~cm})$ on the peripheral axis for all phantoms. About the central axis, there were no significant differences between the cumulative 554
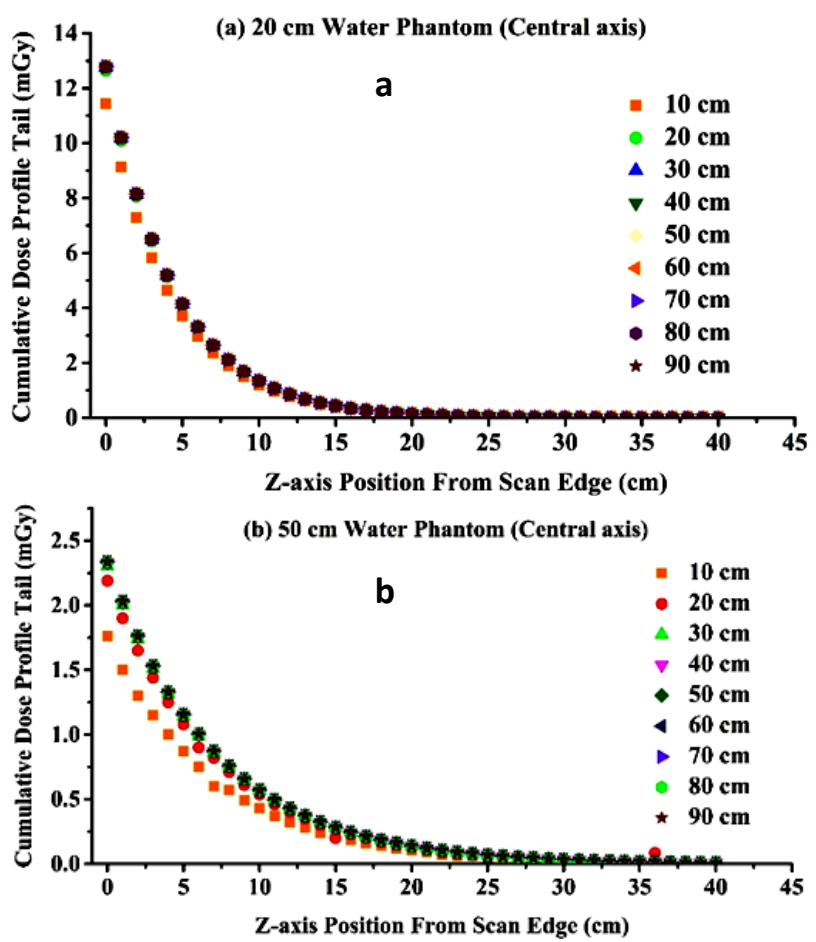

Figure 3. Cumulative dose profile tails beyond the scan lengths of $10-90 \mathrm{~cm}$ on the central axis for (a) the $20 \mathrm{~cm}$ phantom and (b) $50 \mathrm{~cm}$ water phantom.

dose profile tails after approaching the scan length of $30 \mathrm{~cm}$ for all phantoms (maximum difference $<1 \%$ average on all phantom diameters). Maximum differences between the lowest and largest scan length, i.e. $10 \mathrm{~cm}$ and 90 $\mathrm{cm}$ on the central axis are $24 \%$ and $7 \%$ for $50 \mathrm{~cm}$ and $16 \mathrm{~cm}$ phantoms respectively.

Due to the low dependency of the cumulative dose profile tails on the scan lengths larger than $30 \mathrm{~cm}$, the tail of scan length of $30 \mathrm{~cm}$ was Int. J. Radiat. Res., Vol. 19 No. 3, July 2021 
analyzed in detail as shown in figure $5(\mathrm{a}, \mathrm{b})$ in a semi-logarithmic scale on the central and peripheral axes for $16-55 \mathrm{~cm}$ water phantoms. The reason for the minus values in the graphs is that the cumulative dose profile tails have values lower than unity for points far from the scan edge.
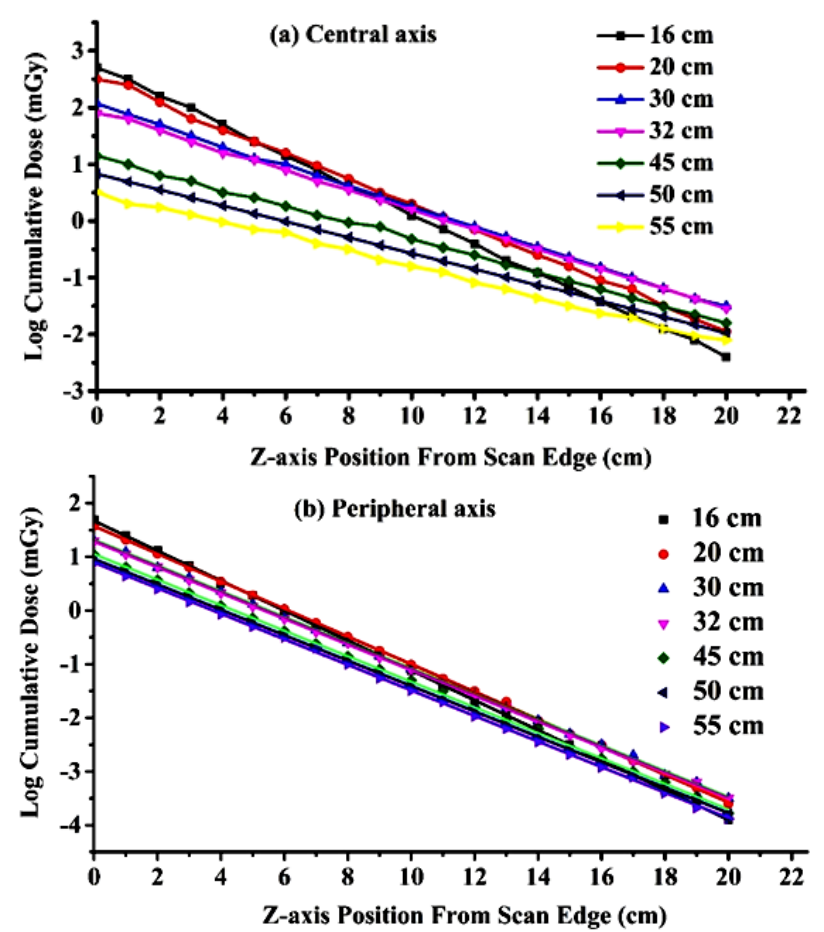

Figure 5. The cumulative dose profile tails beyond the scan length of $30 \mathrm{~cm}$ on the (a) central and (b) peripheral axis of the $16-55 \mathrm{~cm}$ water phantoms in the semi-logarithmic scale.

The straight lines in the semi-logarithmic scale in figure 5 show the exponential falloff estimated by equation 3 with a decreasing diameter-dependent slope (with the average R-square $>0.99$ ).

$\mathrm{D}(\mathrm{z})=A \mathrm{e}^{-\mathrm{Bz}}$

Where D (mGy) is the absorbed dose at each point along the central or peripheral axis; $\mathrm{z}(\mathrm{cm})$ is the distance from the scan edge, $\mathrm{A}$ ( $\mathrm{mGy}$ ) and B $\left(\mathrm{cm}^{-1}\right)$ are constant coefficients for each phantom diameter as shown in table 3 .
Table 3. The A ( $\mathrm{mGy}$ ) and B $\left(\mathrm{cm}^{-1}\right)$ coefficients for the central and peripheral axes for different water phantom sizes.

\begin{tabular}{|c|c|c|c|c|}
\hline \multirow{2}{*}{$\begin{array}{c}\text { Phantom } \\
\text { Diameter } \\
(\mathbf{c m})\end{array}$} & \multicolumn{2}{|c|}{ Central axis } & \multicolumn{2}{c|}{ Peripheral axis } \\
\cline { 2 - 5 } & $\mathrm{A}$ & $\mathrm{B}$ & $\mathrm{A}$ & $\mathrm{B}$ \\
\hline $\mathbf{1 0}$ & 15.21 & 0.343 & 6.91 & 0.344 \\
\hline $\mathbf{1 5}$ & 14.32 & 0.266 & 5.77 & 0.280 \\
\hline $\mathbf{2 0}$ & 12.37 & 0.225 & 4.79 & 0.250 \\
\hline $\mathbf{2 5}$ & 10.28 & 0.199 & 4.69 & 0.248 \\
\hline $\mathbf{3 0}$ & 7.73 & 0.181 & 3.21 & 0.241 \\
\hline $\mathbf{3 5}$ & 5.62 & 0.167 & 3.12 & 0.240 \\
\hline $\mathbf{4 0}$ & 4.64 & 0.157 & 3.10 & 0.239 \\
\hline $\mathbf{4 5}$ & 3.56 & 0.148 & 2.63 & 0.238 \\
\hline $\mathbf{5 0}$ & 2.70 & 0.141 & 2.41 & 0.238 \\
\hline & & & & \\
\hline
\end{tabular}

\section{DISCUSSION}

A detailed analysis of cumulative dose profile tail beyond the scan range in CT examinations was performed in this study by using a validated MC model of a typical CT scanner, DSFs, and the well-known convolution method.

At the first step, the shape, trend, and extension of the DSFs, especially their scatter component, considering the phantom size was analyzed (figure 2). The results show the relatively lower amplitude and extension of the scatter component of DSFs in the peripheral axis compared to the central axis and also in small phantoms compared to the large phantoms. The same trends have been reported in the literature (4). The reason for these observations may be related to the amount of material in the pass of the primary beam which affects the scatter probability (3). The more material in the pass of the beam, the lower falloff speed, the longer range extension, i.e. the higher full width at tenth maximum (FWTM) and full width at half maximum (FWHM) of the scatter component of DSFs. The central to the peripheral ratio of the FWTM increases from 1.17 to 2.35 as the phantom diameter increases. On the other hand, as expected, the peripheral to the central ratio of the maximum amplitude (at $\mathrm{z}=0$ ) increases from 
1.29 to 21.96 with increasing the phantom size.

In the second step, it was found that the trend of the cumulative dose profile tail beyond the scan range according to the phantom diameter, scan length, and radial position (central and peripheral axes) was similar to the scatter component of DSFs. An interesting result observed from figure 5 is the extension of the cumulative dose profile tail up to about $40 \mathrm{~cm}$ from the scan edge which shows the importance of dose received to the points far from the scan edge ${ }^{9}$. . However, there is a position along the z-axis after which the dose can be considered negligible. This position is where the amplitude of the cumulative dose profile tail reduces to its tenth value at the edge. For the central axis, it is $34 \mathrm{~cm}$ and $18 \mathrm{~cm}$ for the largest $(50 \mathrm{~cm})$ and smallest $(16 \mathrm{~cm})$ phantom respectively, and for the peripheral axis, it is around $29 \mathrm{~cm}$ for all phantoms. These results confirm the increasing trend in FWTM obtained in this study and also reported in the literature for the cumulative dose profile with increasing phantom size (11).

The low dependency of cumulative dose profile tail on the scan lengths of $10-90 \mathrm{~cm}$ was confirmed by statistical analysis. This phenomenon can be explained by the equilibrium dose and equilibrium length concepts. Equilibrium dose is the cumulative dose at the center of enough long scan length, i.e. equilibrium length, after which the scatter tail of enough far dose profiles have no significant effect on the cumulative dose at the center of the scan length (7). Similarly, at the edge of the scan range after approaching a threshold scan length, the scatter tail of enough far dose profiles located inside and around the center of the scan range have no significant effect in increasing the dose at the edge. The threshold occurs in longer scan lengths on the central axis compared to the peripheral axis due to more scatter tail extension on the central axis.

The decreasing trend of the cumulative dose profile tails was modeled by an exponential equation with dedicated $A$ and $B$ coefficients for each phantom size. The B coefficient, i.e. the slope of the graphs in figure 5 , decreases from $0.343 \mathrm{~cm}^{-1}$ to $0.141 \mathrm{~cm}^{-1}$ and $0.344 \mathrm{~cm}^{-1}$ to 0.238 $\mathrm{cm}^{-1}$ by increasing phantom diameter respectively for the central and peripheral axes since the scatter component of DSF becomes wider (higher FWTM) as the phantom diameter increases. The overall effect in the convolution process is higher scatter buildup amplitude at all points which causes the lower falloff slope for large phantoms. The A coefficient, i.e. the y-intercept of the lines, decreases from 15.21 mGy to $2.7 \mathrm{mGy}$ and $6.91 \mathrm{mGy}$ to $2.41 \mathrm{mGy}$ respectively for the central and peripheral axes by increasing the phantom diameter. This trend is due to the higher amplitude of the cumulative dose profile tails at the scan edge for the phantoms with smaller diameters. Equation 3 can be used to calculate the dose received to each point beyond the scan range considering the phantom diameter and independent on the scan length. The results of this study are of interest for investigation on the dose of organs located at the edge and outside of the scan range in body CT examinations.

\section{CONCLUSION}

The amplitude and falloff speed of the cumulative dose profile tail decreases $82 \%$ and $59 \%$ on the central axis and $65 \%$ and $31 \%$ on the peripheral axis by increasing the water phantom diameter. Also, the statistical analysis shows the negligible dependency of cumulative dose profile tail on the scan length. The cumulative dose profile tail has an exponential falloff from the scan edge described by equation 3 for each phantom size. The equation 3 is a simple, quick, and accurate enough way to calculate absorbed dose at any point beyond the scan range for all scan lengths in the body region.

\section{ACKNOWLEDGMENTS}

The Research was carried out under the current budget of the Nuclear Engineering Department, Shiraz University.

Conflicts of interest: Declared none.

Int. J. Radiat. Res., Vol. 19 No. 3, July 2021 


\section{REFERENCES}

1. Tack D and Gevenois PA (2007) Radiation dose from adult and pediatric multidetector computed tomography. Berlin, Germany: Springer.

2. Dixon RL (2003) A new look at CT dose measurement: beyond CTDI. Medical physics, 30(6): 1272-80.

3. Boone JM (2007) The trouble with CTDI100. Med Phys, 34 (4):1364-71.

4. Boone JM (2009) Dose spread functions in computed tomography: A Monte Carlo study. Med phys, 36(10): 454754.

5. Turner AC, Zankl M, DeMarco JJ, Cagnon $\mathrm{CH}$, Zhang $\mathrm{D}$, Angel E, Cody DD, Stevens DM, McCollough CH, McNittGray MF (2010) The feasibility of a scanner-independent technique to estimate organ dose from MDCT scans: Using to account for differences between scanners. Med phys, 37(4): 1816-25.

6. Shope TB, Gagne RM, Johnson GC (1981) A method for describing the doses delivered by transmission $\mathrm{x}$-ray computed tomography. Med phys, 8(4): 488-95.

7. American Association of Physicists in Medicine (2010) Comprehensive methodology for the evaluation of radiation dose in X-ray computed tomography. (Task Group 111), 20740-3846.

8. American Association of Physicists in Medicine (2011) Sizespecific dose estimates (SSDE) in pediatric and adult body CT examinations (Task Group 204). College Park, Md: American Association of Physicists in Medicine, 14.

9. Li X, Zhang D, Liu B (2012) Equations for CT dose calculations on axial lines based on the principle of symmetry. Med phys, 39(9): 5347-52.

10. Li X, Zhang D, Liu B (2013) Monte Carlo assessment of CT dose equilibration in PMMA and water cylinders with diameters from 6 to $55 \mathrm{~cm}$. Med Phys, 40(3): 031903.

11. Li X, Zhang D, Liu B (2014) Longitudinal dose distribution and energy absorption in PMMA and water cylinders undergoing CT scans. Med Phys, 41(10): 101912.

12. Jan $S$, Benoit $D$, Becheva $E$, Carlier T, Cassol F, Descourt $P$, Frisson T, Grevillot L, Guigues L, Maigne L, Morel C (2011) GATE V6: a major enhancement of the GATE simulation platform enabling modelling of CT and radiotherapy. Physics in Medicine \& Biology, 56(4): 881.

13. Jan S, Santin G, Strul D, Staelens S, Assie K, Autret D, Avner S, Barbier R, Bardies M, Bloomfield PM, Brasse D (2004) GATE: a simulation toolkit for PET and SPECT. Physics in Medicine \& Biology, 49(19): 4543.

14. Cranley K, Gilmore BJ, Fogarty GW, Desponds L (1997) IPEM Report 78: Catalogue of diagnostic X-ray spectra and other data. York: The Institute of Physics and Engineering in Medicine.

15. Parsi M, Sohrabi M, Mianji F, Paydar R (2017) Gantry angulation effects on $\mathrm{CT}$ dose along the $\mathrm{z}$-axis direction in head examinations. Radiation Protection Dosimetry, 177(4): 458 $-65$.

16. DeMarco JJ, Cagnon CH, Cody DD, Stevens DM, McCollough CH, O'Daniel J, McNitt-Gray MF (2005) A Monte Carlo based method to estimate radiation dose from multidetector CT (MDCT): cylindrical and anthropomorphic phantoms. Physics in Medicine \& Biology, 50(17): 3989.

17. Jarry G (2002) Monte Carlo dose verification of a commercial CT scanner with application for patient specific dosimetry. UCLA.

18. Stamatis DH (2002) Six sigma and beyond: Statistical process control (Vol. 4). CRC Press. United States. 
